Article

\title{
Fabrication of Nanopillar Micropatterns by Hybrid Mask Lithography for Surface-Directed Liquid Flow
}

\author{
Shinya Sakuma *, Masakuni Sugita and Fumihito Arai \\ Department of Micro-Nano Systems Engineering, Nagoya University, Nagoya 464-8603, Japan; \\ E-Mails: masakuni@biorobotics.mech.nagoya-u.ac.jp (M.S.); arai@mech.nagoya-u.ac.jp (F.A.) \\ * Author to whom correspondence should be addressed; \\ E-Mail: sakuma@biorobotics.mech.nagoya-u.ac.jp; \\ Tel.: +81-52-789-5026; Fax: +81-52-789-5027.
}

Received: 19 March 2013; in revised form: 12 May 2013 / Accepted: 23 May 2013 /

Published: 5 June 2013

\begin{abstract}
This paper presents a novel method for fabricating nanopillar micropatterns for surface-directed liquid flows. It employs hybrid mask lithography, which uses a mask consisting of a combination of a photoresist and nanoparticles in the photolithography process. The nanopillar density is controlled by varying the weight ratio of nanoparticles in the composite mask. Hybrid mask lithography was used to fabricate a surface-directed liquid flow. The effect of the surface-directed liquid flow, which was formed by the air-liquid interface due to nanopillar micropatterns, was evaluated, and the results show that the oscillation of microparticles, when the micro-tool was actuated, was dramatically reduced by using a surface-directed liquid flow. Moreover, the target particle was manipulated individually without non-oscillating ambient particles.
\end{abstract}

Keywords: nanopillar; lithography; microrobot; surface-directed liquid flow; microfluidic chip

\section{Introduction}

Cell manipulation in the confined space of a microfluidic chip has contributed in the bioengineering field, because of its benefits of low contamination, repeatability and high throughput ability [1,2]. In particular, microrobots on a chip have great advantages for the treatment of biological cells with high throughput, due to their high speed and high accuracy [3]. Several actuation methods of microrobots 
have been proposed for microscale cell manipulation, such as optical tweezers [4,5], magnetic actuators [6,7] and bubble robots driven by optically induced thermocapillary flow [8]. In previous studies, we proposed magnetically driven micro-tools (MMTs) for on-chip cell manipulation [9-12]. The reason for this is that magnetic fields can be a suitable power source for an on-chip robot, because of their non-contact drive, low invasiveness with respect to a cell and low production cost. Thus, a considerable amount of research has been carried out on magnetic actuators [6,7,9-14]. We have achieved the high speed actuation of MMTs with high output force by applying the magnetic field of horizontally arranged permanent magnets. However, the motion of these micro-tools was transmitted to the ambient cells in a microchannel, due to fluidic oscillations (Figure 1a). This is a serious problem, since it reduces the speed and precision of cell manipulation on a chip.

On the other hand, it is important to control the surface geometry when fabricating functional surfaces, because nanostructures on a surface enhance the characteristics of the surface, such as its friction force [15], wettability [16] and optical resonance [17]. The wettability of a surface can be used to control the surface tension of a liquid and restrict the flow direction in a microfluidic chip $[18,19]$. These surface-directed liquid flows formed by nanopatterned surfaces are, therefore, very important for biomedical applications using microfluidic chips.

Herein, we describe an innovative cell manipulation approach using surface-directed liquid flow. Figure $1 \mathrm{~b}$ shows the conceptual overview of the cell manipulation within surface-directed liquid flow. The MMTs are placed in a microfluidic chip and actuated by permanent magnets placed on the outer motorized stages. An air-liquid interface is utilized to separate the driving component of the micro-tool from the microchannel in order to reduce fluidic oscillation when the MMT is actuated. This air-liquid interface is formed by nanopillar micropatterns that alter the surface wettability. The manipulation method can be applied to a wide range of cell manipulations, because of its high speed and strong output force.

Figure 1. Surface-directed liquid flow for on-chip cell manipulation: (a) conventional microchannel and (b) surface-directed liquid flow.

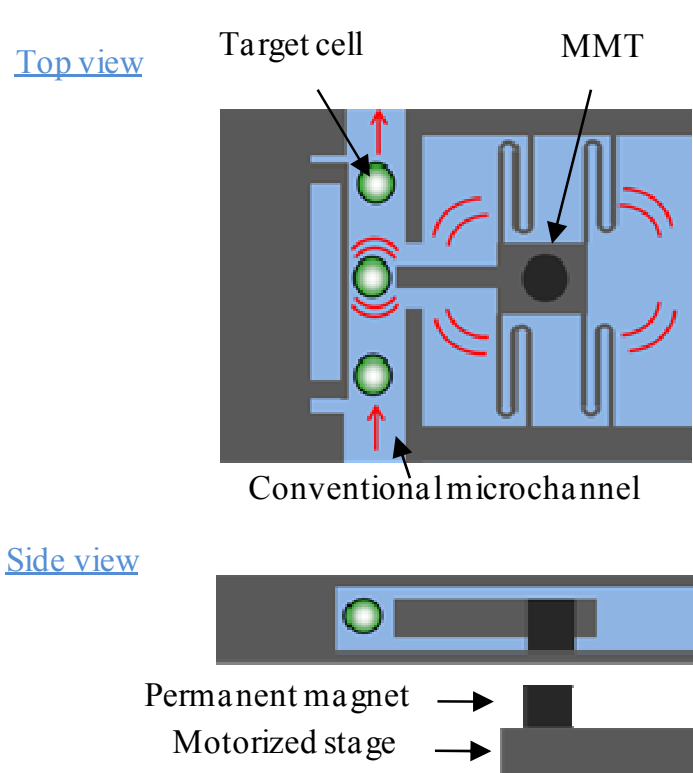

(a)

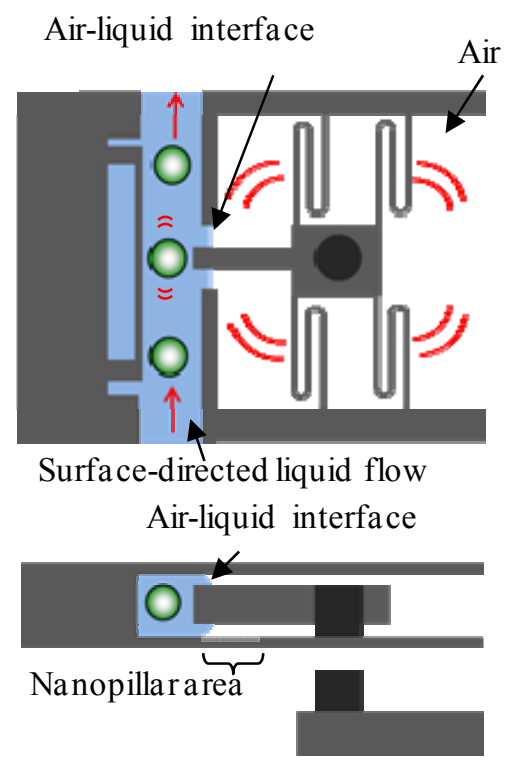

(b) 


\section{Fabrication of Nanopillar Micropatterns}

In this paper, we propose a hybrid mask lithography technique for fabricating nanopillar micropatterns to obtain surface-directed liquid flow.

Previously, several methods were commonly used to fabricate the nanogeometric surface as a nanopillar array, such as by plasma etching, by photolithography, and so on. In terms of the microfluidic chip application, an important parameter of methods for fabricating microfluidic chips is the size of nanopatterned surfaces. Plasma etching and photolithography are generally used to fabricate nanopatterned surfaces containing nanopillar arrays. Reactive ion etching (also known as plasma etching) can be used to fabricate black silicon, which is a needle-like structure produced by using non-optimal etching conditions [20,21]. Its sharp structure produces a hydrophobic surface on a wafer. However, it is difficult to control the nanopillar height and density, because the fabrication process depends on the conditions of the etching and deposition gases. Electron-beam lithography is commonly used to fabricate nanopatterns with dimensions below the diffraction limit of photolithography [22,23]. However, this technique involves scanning an electron beam, so it takes a long time to pattern a large area. Previously, the fabrication method, which utilized the combination of electron-beam and photo-lithographies, was proposed for the microfluidic chip applications [24]. This technique is based on multi-step exposure and can fabricate the microchannel, which has a nanopillar array. However, the technique requires multi-step procedures involving electron-beam lithography, which echoes the technical difficulties. In this paper, we propose a hybrid mask lithography technique that employs a photomask containing nanoparticles as a mask for fabricating nanopillars. Figure 2 depicts the concept of this method. It is based on a simple two-step procedure that involves photolithography and etching, which are standard microfabrication processes. Our lithography method can fabricate nanopillar micropatterns with a diffraction-limited accuracy. In the photolithography process (Figure 2a,b), a composite of positive photoresist and nanoparticles is exposed using the two different masks - the photo mask and the dispensed nanoparticles. The photomask was used to fabricate an arbitrary 2D pattern with a photolithographic resolution, and the nanoparticles were used to fabricate nanodot patterns with a diffraction-limited size. These hybrid etching masks (Figure 2c) for deep reactive ion etching (DRIE) can be used to fabricate microchannel and nanopillar patterns. The nanopillar height could also be controlled as the same height as the micropattern formed on the substrate surface (Figure 2d). Hybrid mask lithography was demonstrated, as shown in Figure 3a. We fabricated nanopillars with uniform heights in the micropattern (Figure 3b). In this case, the DRIE technique was used for etching. In addition, OFPR (Product from Tokyo Ohka Co., Ltd., Kawasaki, Japan) was used as positive photoresist and Fe nanoparticle (Tateyama Machine Co., Ltd., Toyama, Japan) was used as nanoparticles. Micropatterns (including nanopillars) were fabricated. The features of this process are summarized as follows.

(1) It is possible to fabricate arbitrary 2D patterns using photolithography.

(2) Nanopillars at the diffraction-limited size are achieved by using nanoparticles mask.

(3) The density of nanopillars can be controlled by simply changing the weight ratio of the nanoparticles of the composite.

(4) The height of nanopillars can also be controlled to be the same as the micropattern, which is the original surface of the substrate. 
Figure 2. Concept and demonstration of hybrid mask lithography: (a) spin coating of composite; (b) patterning of composite; (c) deep reactive ion etching (DRIE); and (d) removal of composite.

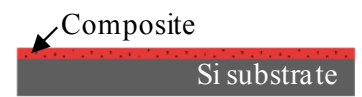

(a)

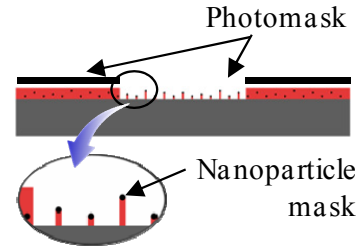

(b)

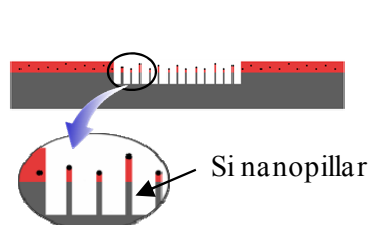

(c)

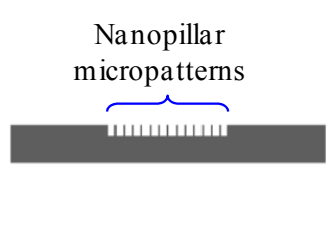

(d)

Figure 3. SEM images of fabricated structure by hybrid mask lithography: (a) perspective view; and (b) cross-sectional image of fabricated nanopillar micropattern.

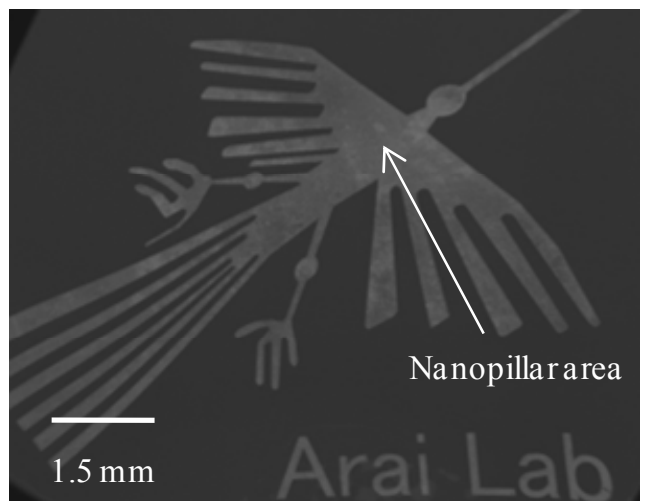

(a)

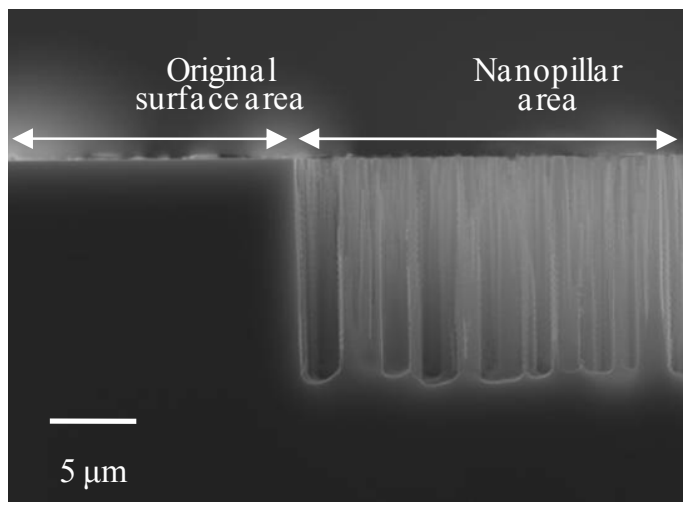

(b)

\section{Results and Discussions}

\subsection{Density Control of Nanopillars}

By using the proposed hybrid mask lithography technique, the nanopillar density could be controlled by simply varying the weight ratio of nanoparticles in the composite. Figure $4 \mathrm{a}-\mathrm{c}$ show a typical top-view of scanning electron microscopy (SEM) images of a region containing nanopillars. Figure 4d,e show a plot of the nanopillar density and the nanopillar diameter as a function of the weight ratio of nanoparticles, respectively. These values were measured by counting the pixels in the SEM image. In these figures, the vertical axis represents the nanopillar density and the nanopillar diameter, while the horizontal axis indicates the weight ratio of nanoparticles. There is a relatively linear relationship between the nanopillar density and the nanoparticle density, when the weight ratio is less than $0.40 \%$. The uniformity of the nanopillars also depends on the uniformity of the nanoparticles. Moreover, the diameters of the nanopillar increases when the weight ratio increases. Significant nanoparticle agglomeration occurred when the weight ratio exceeded $0.50 \%$, and the agglomeration prevented formation of uniform nanopillars. These results indicate that the nanopillar diameter is dependent on the agglomeration of the nanoparticle in a composite. Therefore, the nanopillar density could be simply controlled between 0.0 and about 0.6 pillars per $\mu \mathrm{m}^{2}$ by varying the nanoparticle weight ratio. This technique can be used to control the surface wettability, since it depends on the nanopillar density. 
Figure 4. SEM images of the top of the nanopillar and measured density of the nanopillar as a function of the weight ratio of the nanoparticle. (a) $0.10 \%$; (b) $0.20 \%$; (c) $0.40 \%$; (d) measured density of the nanopillar of the fabricated chip; and (e) measured diameter of the nanopillar of the fabricated chip.

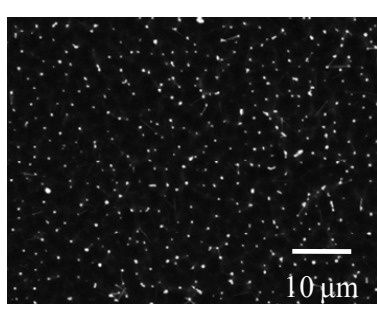

(a)

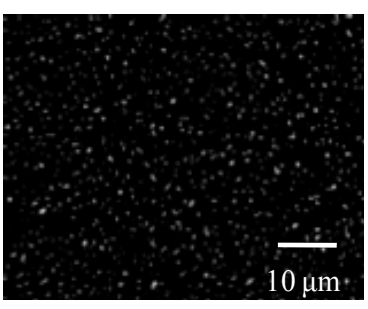

(b)

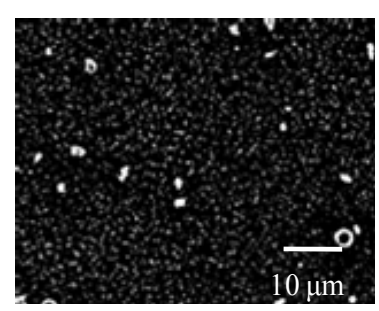

(c)

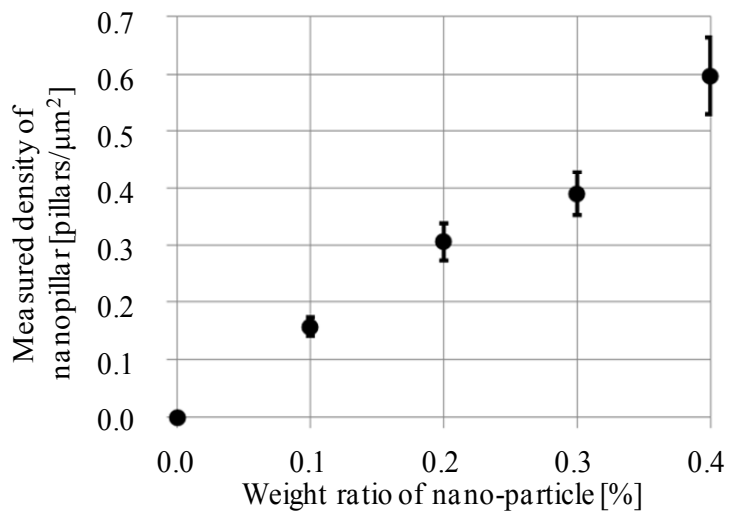

(d)

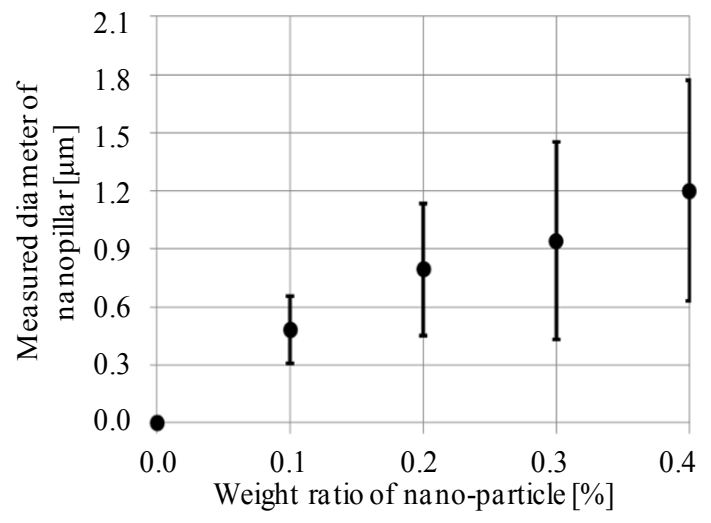

(e)

\subsection{Contact Angle Control}

The benefits of this hybrid mask lithography technique will contribute to the control of the wettability of the surface, which depends on the density of nanopillars.

In order to predict the effect of the nanopillars on the surface wettability, the contact angle was calculated using the same manner as in reference [25]. The nanopillars fabricated by hybrid mask lithography exhibit a unique geometric feature. The Si nanopillars were coated with a fluorocarbon, because $\mathrm{C}_{4} \mathrm{~F}_{8}$ gas was utilized as the passivation gas in the DRIE process. We considered this hybrid geometric feature in an analytical model.

Figure 5a shows the analytical model of the nanopattern. To analyze the contact angle, $S$ is defined as the projected area. For a surface composed of $N$ materials, the total nanopatterned surface is subdivided into $N$ elemental areas, $S_{k}$, whose characteristics vary according to the original materials. The effect of the fraction of each material $\left(f_{k}\right)$ is expressed by Equation (1).

$$
f_{k}=S_{k} / S(k=1,2, \ldots, N)
$$

This equation implies that $f_{k}$ depends on the contribution of each material and the fractional area of each material. In this case, the surface contact angle $(\theta)$ is given by:

$$
\cos \theta=\sum_{k=1}^{N} f_{k} \cos \theta_{k}
$$


where $\theta_{k}$ is the initial contact angle of each material $S_{k}$. In this analytical model, the nanopatterned surface was subdivided into three surfaces: the tops of the pillars (surface A), the sides of the pillars (surface B) and the substrate (surface C). The fractions of these surfaces are given by:

$$
\begin{gathered}
f_{A}=S_{A} / S=\pi d^{2} \rho / 4 \\
f_{B}=S_{B} / S=\pi d h \rho \\
f_{C}=S_{C} / S=1-\pi d^{2} \rho / 4
\end{gathered}
$$

Finally, the contact angle can be calculated using:

$$
\cos \theta=\frac{\pi d^{2} \rho}{4} \cos \theta_{A}+\pi d h \rho \cos \theta_{B}+\left(1-\frac{\pi d^{2} \rho}{4}\right) \cos \theta_{C}
$$

Figure $5 \mathrm{~b}$ shows the contact angles for different nanopillar density. In order to realize greater wettability variation for different nanoparticle densities, a fluorocarbon or $\mathrm{SiO}_{2}$ was deposited on the nanopillars. The height and diameter of the fabricated nanopillars were evaluated from SEM images in order to calculate the contact angle. The measured average of height of nanopillars were $3 \mu \mathrm{m}$, and the standard deviation was $0.2 \mu \mathrm{m}$. The squares indicate the experimental values, while the lines indicate the calculated values (continuous line: Cassie-Baxter model; broken line: Wenzel model). The experimental values were measured from charge coupled device (CCD) images, while the analytical model was calculated using Equation (6). There is close agreement between the experimental values and the values calculated by the Wenzel model in the case of the nanopillar surface, which is obtained after the lift-off process (Figure 2d). As for the coated fluorocarbon data, the experimental values are close to the calculated values by the Wenzel model when the density of nanopillars is more than 0.2 pillars per $\mu \mathrm{m}^{2}$. When the density is from 0.2 to 0.4 pillars per $\mu \mathrm{m}^{2}$, the experimental values disagree with the calculated values. The experimental values are close to the calculated values by Cassie-Baxter model when the density is more than 0.4 pillars per $\mu \mathrm{m}^{2}$. The results indicate that the model shifts from the Wenzel model to the Cassie-Baxter model with increasing of the density of pillars, though our analytical model could not make this point clear. In the case of the $\mathrm{SiO}_{2}$ coated nanopillar, the contact angle is close to the values calculated by the Wenzel model when the nanopillar density is less than 0.1 pillars per $\mu \mathrm{m}^{2}$. When the density is more than 0.1 pillars per $\mu \mathrm{m}^{2}$, the contact angle is approximately $0^{\circ}$. These results show that the contact angle can be controlled between about $120^{\circ}$ and $150^{\circ}$ by simply varying the nanopillar density. Moreover, the contact angle varied between about $110^{\circ}$ and $160^{\circ}$ and about $0^{\circ}$ and $40^{\circ}$ for fluorocarbon and $\mathrm{SiO}_{2}$ deposition, respectively. Therefore, it is possible to control the contact angle by simply varying the nanopillar density and by using suitable coating materials. The wettability was controlled over a wide range, enabling both hydrophobic and hydrophilic surfaces to be obtained. 
Figure 5. Evaluation of the contact angle: (a) analytical model of contact angle and (b) measured contact angle of water droplet as a function of the density of the nanopillars and the photographs of the droplet of the typical results.

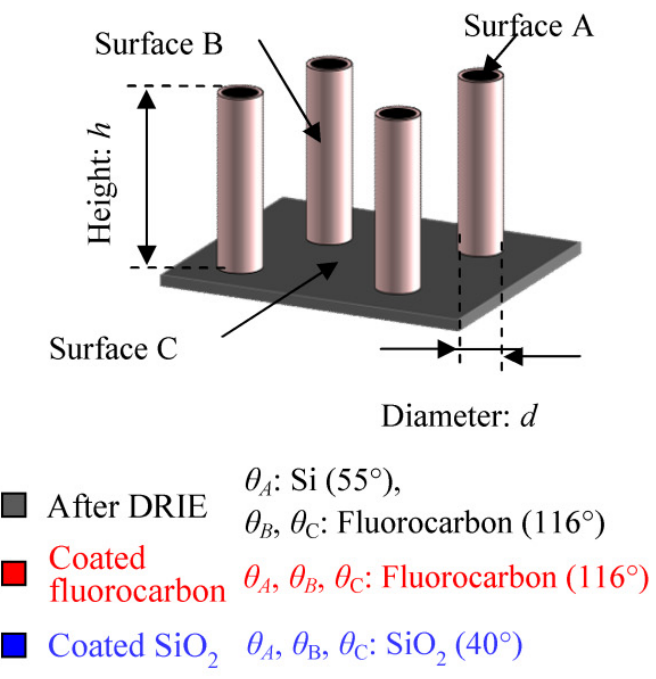

(a)

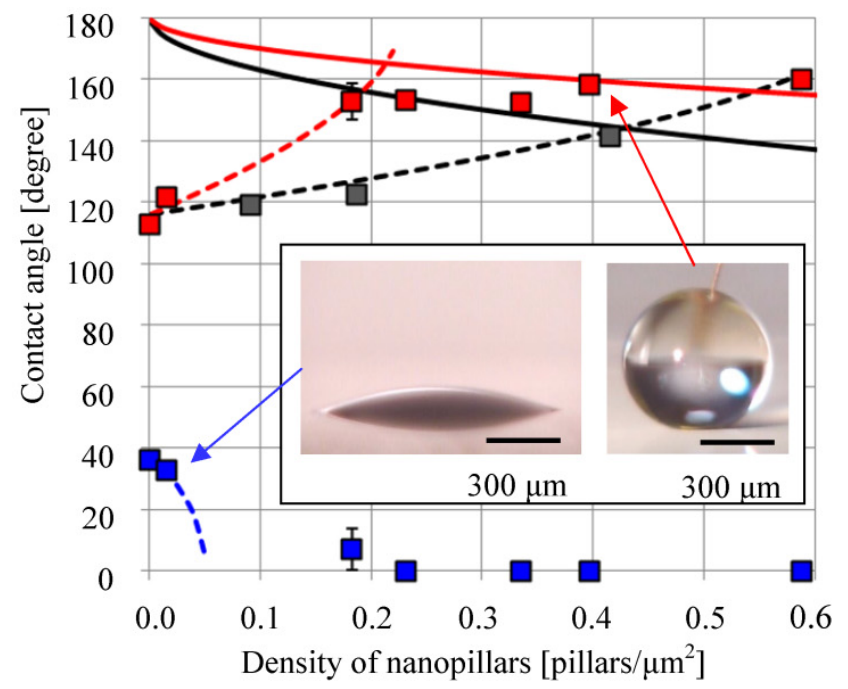

(b)

\subsection{Surface-Directed Liquid Flow}

A surface-directed liquid flow was demonstrated by forming an air-liquid interface. To obtain a hydrophobic surface, the composite containing a $0.4 \%$ weight ratio of nanoparticles was used, and a fluorocarbon was deposited on the nanopillars by a lift-off process (Figure 6a-c) after performing the process shown in Figure 1d. A microfluidic chip was then packaged with spacer and cover layers (Figure 6d). The spacer layer was fabricated using polydimethylsiloxane (PDMS), whose original contact angle was $94^{\circ}$, by the molding technique. The thickness was designed as $250 \mu \mathrm{m}$, because the MMT is $200 \mu \mathrm{m}$. In the fabrication of the cover layer, fluorocarbon was deposited on the glass substrate by the plasma deposition technique.

Figure 6. Fabrication of surface-directed liquid flow. (a) Patterning of OFPR as the sacrifice layer, (b) deposition of fluorocarbon, (c) removal of OFPR and (d) packaging and introduction of water.

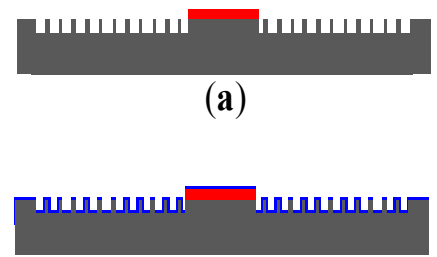

(b)

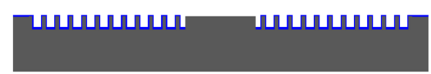

(c)

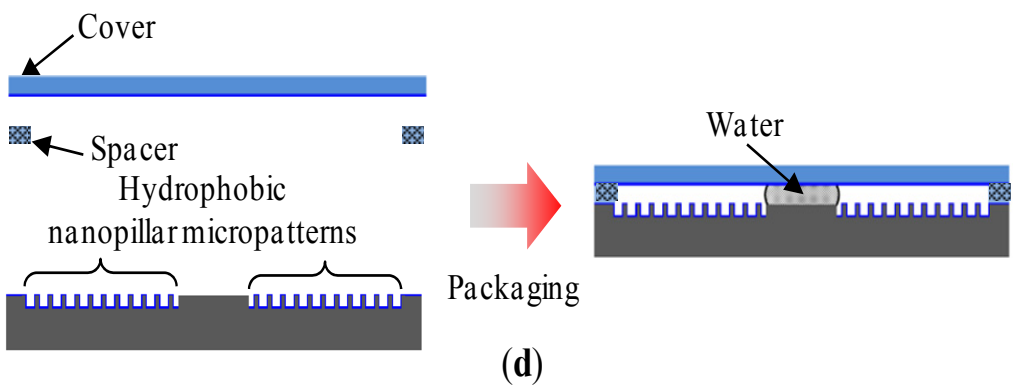

(d)

- $\mathrm{Si}$ OFPR Fluorocarbon - Glass Polydimethylsiloxane 
The fabricated microfluidic chip was demonstrated, as shown in Figure 7. When water was introduced to the microchannel, an air-liquid interface formed along the edge of the micropattern, indicating that we realized a surface-directed liquid flow (Figure 7a). Then, microparticles with a diameter of $20 \mu \mathrm{m}$ were transported at a maximum speed of $2.4 \mathrm{~mm} / \mathrm{s}$ (Figure 7b,c).

Figure 7. Demonstration of surface-directed liquid flow. (a) Introduction of water, (b) forming of air-liquid interface and (c) transportation of microparticles.

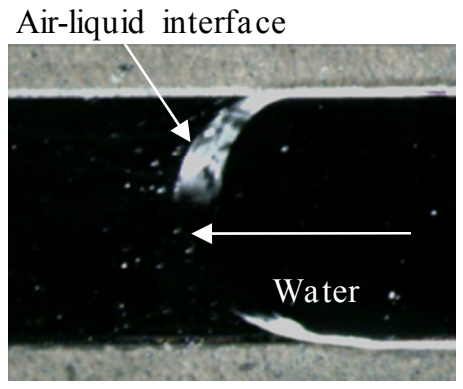

(a)

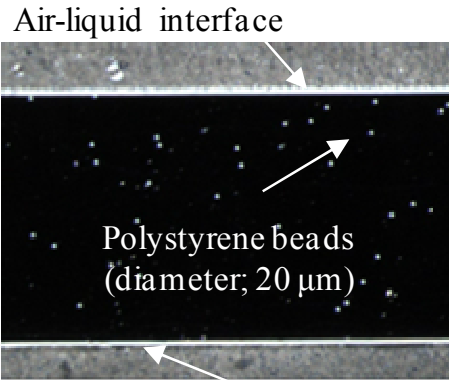

Air-liquid interface

(b)

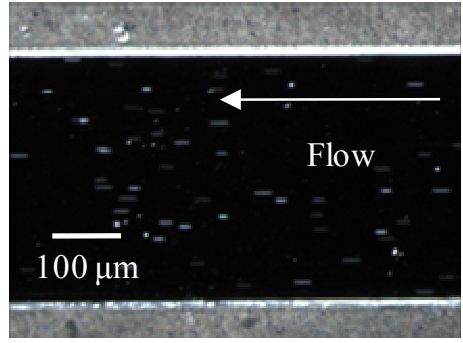

(c)

The effect of the surface-directed liquid flow was evaluated. Figure 8 shows the typical results of the high speed manipulation of microparticles. An MMT was actuated in the microfluidic chip (frequency: $7 \mathrm{~Hz}$; amplitude: $800 \mu \mathrm{m}$ ) in a conventional microchannel (Figure 8a) and a surface directed liquid flow (Figure 8b). The air-liquid interface was stably maintained even when the MMT probe tip was inserted into the microchannel. Moreover, oscillation of the ambient particle was remarkably lower than that for a conventional microchannel. Figure 9 shows the measurement results of the effect of the conventional microchannel (Figure 9a) and the surface-directed liquid flow (Figure 9b) in terms of the microparticles oscillation. The horizontal axis represents the velocity of the micro-tools, while the vertical axis shows the velocity of the oscillation of the microparticle. Each line represents the distance between the micro-tool and the microparticles. In the case of the conventional microchannel, the oscillation of the microparticles was increased versus the speed of the micro-tools or the distance between the micro-tool and the microparticles. In contrast, the oscillation of the microparticle was dramatically reduced in the case of surface-directed liquid flow. Moreover, the target particle was manipulated individually without non-oscillation ambient particles, as shown in Figure 8b. Eventually, we confirmed that the surface-directed liquid flow fabricated by hybrid mask lithography contributed to the on-chip high speed manipulation of a cell. 
Figure 8. Photographs of insertion of a probe tip of magnetically driven micro-tools (MMTs) into (a) the previous microchannel and (b) surface-directed liquid flow.
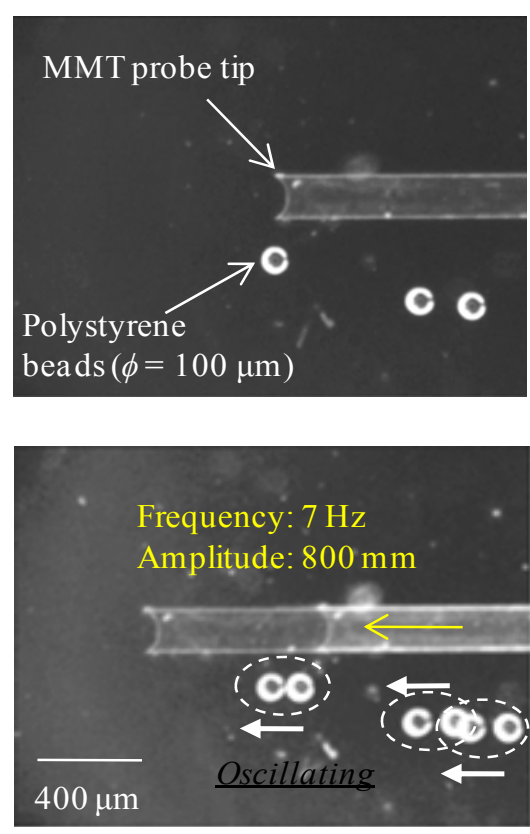

(a)
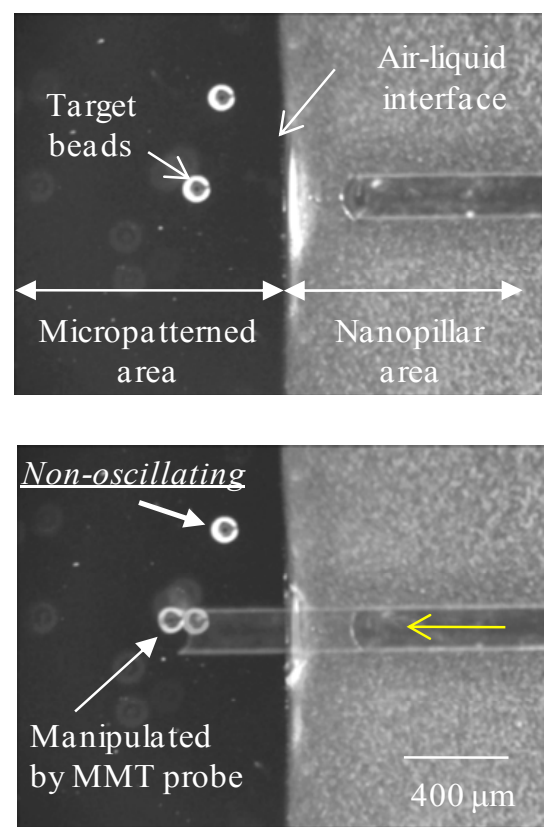

(b)

Figure 9. Measured oscillation velocity of the microparticle. (a) Previous microchannel and (b) surface-directed liquid flow.

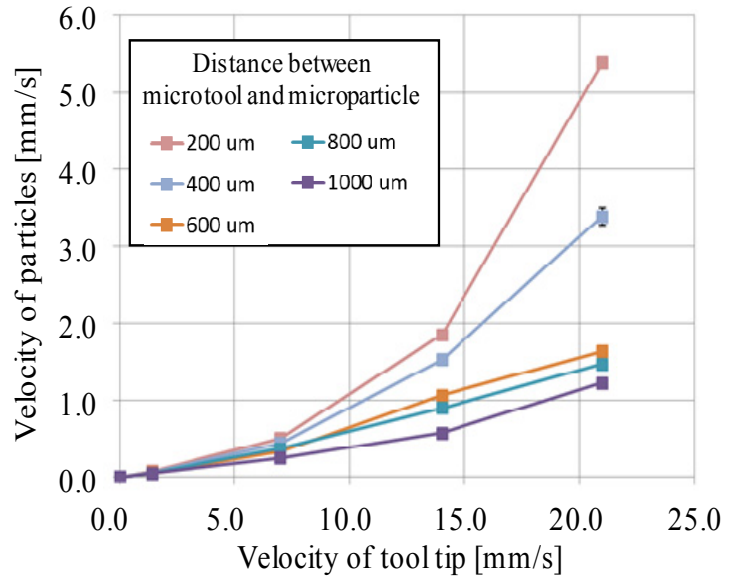

(a)

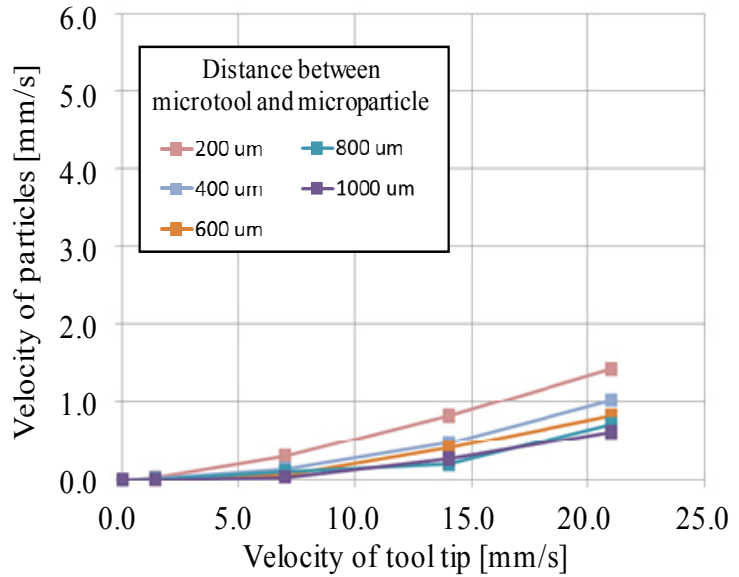

(b)

\section{Conclusions}

In this paper, we proposed hybrid mask lithography for fabricating the nanopillar micropatterns toward microfluidic chip applications. The technique utilized the composite of a positive photoresist and nanoparticles. Hybrid mask lithography was demonstrated, and nanopillar micropatterns were obtained with uniform heights in the original surface of the substrate.

By using this technique, the nanopillar density was controlled between 0.0 and about 0.6 pillars per $\mu \mathrm{m}^{2}$ by simply changing the weight ratio of nanoparticles in the composite. In addition to this advantage, the contact angle of the surface was also controlled between about $120^{\circ}$ and $150^{\circ}$ by changing the density of nanopillars. Moreover, a fluorocarbon or $\mathrm{SiO}_{2}$ was deposited on the 
nanopillars to realize greater wettability variation for different nanoparticle density. The contact angle varied between about $110^{\circ}$ and $160^{\circ}$ and about $0^{\circ}$ and $40^{\circ}$ for fluorocarbon and $\mathrm{SiO}_{2}$ deposition, respectively. The analytical values were calculated using the Cassie-Baxter and Wenzel models, and there are good agreements between the experimental values. From these results, we confirmed that the wettability was controlled over a wide range, enabling both hydrophobic and hydrophilic surfaces to be obtained.

A surface-directed liquid flow was demonstrated by forming an air-liquid interface using the wettability variation. The effect of the surface-directed liquid flow was evaluated. The results show that the oscillation of microparticles, when the micro-tool was actuated, was dramatically reduced by using a surface-directed liquid flow. Moreover, the target particle was manipulated individually without non-oscillation ambient particles. These results indicated that the surface-directed liquid flow fabricated by hybrid mask lithography contributed to the on-chip high speed manipulation of the cell.

The proposed method will be one of the promising techniques to fabricate multi-scale structures for biomedical application. Unlike conventional fabrication techniques, hybrid mask lithography is based on a standard microfabrication process at the wafer level size. Moreover, the proposed method can be applied to cell manipulations in the microfluidic chip.

\section{Acknowledgments}

This work is supported by a Grant-in-Aid for Scientific Research from the Ministry of Education, Culture, Sports, Science and Technology (23106002) and also the Japan Society for the Promotion of Science.

\section{References}

1. Wakayama, T.; Perry, A.C.F.; Zuccotti, M.; Johnson, K.R.; Yanagimachi, R. Full-term development of mice fromenucleated oocytes injected with cumulus cell nuclei. Nature 1998, 394, 369-374.

2. Sun, Y.; Wan, K.T.; Roberts, K.P.; Bischof, J.C.; Nelson, B.J. Mechanical property characterization of mouse zona pellucida. IEEE Trans. NanoBiosci. 2003, 2, 279-286.

3. Trimmer, W.S.N. Microrobots and micromechanical systems. Sens. Actuators 1989, 19, 267-287.

4. Arai, F.; Ogawa, M.; Fukuda, T. Selective manipulation of a microbe in a microchannel using a teleoperated laser scanning manipulator and dielectrophoresis. Adv. Rob. 1999, 13, 343-345.

5. Onda, K.; Arai, F. Multi-beam bilateral teleoperation of holographic optical tweezers accelerated by general-purpose GPU. Opt. Express 2012, 20, 3642-3653.

6. Mensing, G.A.; Pearce, T.M.; Graham, M.D.; Beebe, D.J. An externally driven magnetic microstirrer. Philos. Trans. R. Soc. Lond. Ser. A 2004, 362, 1059-1068.

7. Ryu, K.S.; Shaikh, K.; Goluch, E.; Fan, Z.; Liu, C. Micro magnetic stir-bar mixer integrated with parylene microfluidic channels. Lab Chip 2004, 4, 608-613.

8. Hu, W.; Ishii, K.S.; Ohta, A.T. Micro-assembly using optically controlled bubble microrobots. Appl. Phys. Lett. 2011, 99, 094103, doi:10.1063/1.3631662.

9. Yamanishi, Y.; Sakuma, S.; Onda, K.; Arai, F. Powerful actuation of magnetized microtools by focused magnetic field for particle sorting in a chip. Biomed. Microdevices 2008, 10, 411-419. 
10. Yamanishi, Y.; Sakuma, S.; Kihara, Y.; Arai, F. Fabrication and application of 3-D magnetically driven microtools. J. Microelectromechanical Sys. 2010, 19, 350-357.

11. Hagiwara, M.; Kawahara, T.; Yamanishi, Y.; Masuda, T.; Feng, L.; Arai, F. On-chip magnetically actuated robot with ultrasonic vibration for single cell manipulations. Lab Chip 2011, 11, 2049-2054.

12. Hagiwara, M.; Kawahara, T.; Yamanishi, Y.; Arai, F. Precise control of magnetically driven microtools for enucleation of oocytes in a microfluidic chip. Adv. Rob. 2011, 25, 991-1005.

13. Atencia, J.; Beebe, D.J. Magnetically-driven biomimetic micro pumping using vortices. Lab Chip 2004, 4, 598-602.

14. Gassner, A.-L.; Abonnenc, M.; Chen, H.-X.; Morandini, J.; Josserand, J.; Rossier, J.S.; Busnel, J.-M.; Girault, H.H. Magnetic forces produced by rectangular permanent magnets in static microsystems. Lab Chip 2009, 9, 2356-2363.

15. Stubenrauch, M.; Fischer, M.; Kremin, C.; Stoebenau, S.; Albrecht, A.; Nagel, O. Black silicon-new functionalities in microsystems. J. Micromech. Microeng. 2006, 16, S82-S87.

16. Onda, T.; Shibuichi, S.; Satoh, N.; Tsujii, K. Super-water-repellent fractal surfaces. Langmuir 1996, 12, 2125-2127.

17. Walker, B.N.; Stolee, J.A.; Pickel, D.L.; Retterer, S.T.; Vertes, A. Tailored silicon nanopost arrays for resonant nanophotonic ion production. J. Phys. Chem. C 2010, 114, 4835-4840.

18. Bouaidat, S.; Hansen, O.; Bruus, H.; Berendsen, C.; Bau-Madsen, N.K.; Thomsen, P.; Wolff, A.; Jonsmann, J. Surface-directed capillary system; theory, experiments and applications. Lab Chip 2005, 5, 827-836.

19. Hong, L.; Pan, T. Surface microfluidics fabricated by photopatternable superhydrophobic nanocomposite. Microfluid. Nanofluid. 2011, 10, 991-997.

20. Jansen, H.; Boer, M.; Legtenberg, R.; Elwenspoek, M. The black silicon method: A universal method for determining the parameter setting of a fluorine-based reactive ion etcher in deep silicon trench etching with profile control. J. Micromech. Microeng. 1995, 5, 115-120.

21. Choi, C.H.; Kim, C.J. Large slip of aqueous liquid flow over a nanoengineered superhydrophobic surface. Phys. Rev. Lett. 2006, 96, 066001, doi:10.1103/PhysRevLett.96.066001.

22. Martines, E.; Seunarine, K.; Morgan, H.; Gadegaard, N.; Wilkinson, C.D.W.; Riehle, M.O. Superhydrophobicity and superhydrophilicity of regular nanopatterns. Nano Lett. 2005, 5, 2097-2103.

23. Lee, H.; Lee, B.P.; Messersmith, P.B. A reversible wet/dry adhesive inspired by mussels and geckos. Nature 2007, 448, 338-341.

24. Choi, C.K.; Fowlkes, J.D.; Retterer, S.T.; Siuti, P.; Lyer, S.; Doktycz, M.J. Surface charge- and space-dependent transport of proteins in crowded environments of nanotailored posts. ACS Nano 2010, 4, 3345-3355.

25. Hazlett, R.D. Fractal applications: Wettability and contact angle. J. Colloid Interface Sci. 1990, 137, 527-533.

(C) 2013 by the authors; licensee MDPI, Basel, Switzerland. This article is an open access article distributed under the terms and conditions of the Creative Commons Attribution license (http://creativecommons.org/licenses/by/3.0/). 